\title{
Parallel Factor Analysis for multidimensional decomposition of fNIRS data - A validation study
}

\author{
Hüsser, Alejandra1, a, b, Caron-Desrochers, Laura', a, b, Tremblay, Julie ${ }^{\text {a }}$, Vannasing, \\ Phetsamone $^{a}$, Martínez-Montes, Eduardo ${ }^{\mathrm{c}, \dagger}$ \& Gallagher, Anne ${ }^{\mathrm{a}, \mathrm{b}, \dot{\dagger},{ }^{*} \text { * }}$
}

${ }^{a}$ Center of the Sainte-Justine University Hospital Center, Neurodevelopmental Optical Imaging Laboratory (LIONlab), Research, 3175 Chemin de la Côte-Sainte-Catherine, Montreal QC, Canada, H3T 1C5

${ }^{\mathrm{b}}$ Université de Montréal, Department of Psychology, Pavillon Marie-Victorin, P.O. Box 6128 Centre-ville Station, 2900 Boulevard Édouard-Montpetit, Montreal QC, Canada, QC H3T 1J4

${ }^{\mathrm{c} C u b a n}$ Neurosciences Center (CNEURO), Street 190 e / 25 and 27 Cubanacan, Playa Havana, CP 11600, Cuba

${ }^{1}$ Co-First author: authors contributed equally to this study.

${ }^{\dagger} \mathrm{Co}-\mathrm{Senior}$ author: equal contribution as co-senior authors.

*Anne Gallagher, E-Mail: anne.gallagher@umontreal.ca

\begin{abstract}
Significance: Functional near-infrared spectroscopy (fNIRS) is a neuroimaging technique that uses nearinfrared lights to estimate cerebral hemodynamic response, based on concentration changes in oxygenated and deoxygenated hemoglobin. A multi-dimensional decomposition technique, parallel factor (PARAFAC) analysis, has been validated for the identification of artifacts and cerebral activation patterns in electroencephalography and neuroimaging. Aim: We aimed at introducing and validating the use of the PARAFAC model for fNIRS data analysis, which is inherently multidimensional (time, space, wavelength). Approach: Eighteen healthy adults underwent fNIRS acquisition during a verbal fluency task. The signal-to-noise ratio and Pearson's correlation were used to evaluate the efficacy of PARAFAC for motion artifact correction. Temporal, spatial and hemodynamic characteristics of the PARAFAC component allowed to identify task-related cerebral activations. Results: Motion artifact correction with PARAFAC led to significant improvements in data quality and other advantages as compared to traditional 2D approaches (ICA, tPCA). Although PARAFAC revealed a slightly more distributed functional network, temporal and spatial characteristics of the task-related brain activation identified with PARAFAC mostly overlapped with those obtained with commonly used approaches. Conclusion: This study describes the first implementation of PARAFAC in fNIRS and supports it as a promising data-driven alternative for multi-dimensional data analyses in fNIRS.
\end{abstract}

Keywords: Near-infrared spectroscopy, multidimensional decomposition, parallel factor analysis (PARAFAC), canonical decomposition, artifact correction, language paradigm.

\section{Introduction}

Functional near-infrared spectroscopy (fNIRS) is a non-invasive neuroimaging technique that uses light of at least two different wavelengths in the near-infrared spectrum in order to assess brain activity based on neurovascular coupling. The specific absorption properties of oxygenated $(\mathrm{HbO})$ and deoxygenated $(\mathrm{HbR})$ hemoglobin allow individual assessments of concentration changes in both $\mathrm{HbO}$ and $\mathrm{HbR}$ separately ${ }^{1}$. Thus, data can be interpreted not only for temporal and spatial characteristics, but also for the wavelength or hemodynamic domain. Compared to functional magnetic resonance imaging (fMRI), fNIRS offers lower spatial resolution but allows a more comprehensive representation of the hemodynamic response, and at a faster time resolution $(\approx 0.1 \mathrm{~s})$. Currently, many of the fNIRS data analysis approaches (e.g. wavelet filtering, decomposition, spline interpolation, etc.) do not take fully into account the multidimensional structure of the fNIRS signal and are limited to two dimensions, often considering either time and 
space, or time and wavelength ${ }^{2-4}$. Two-dimensional (2D) analyses require that data with more dimensions, such as fNIRS data, undergo superficial unfolding before processing, for example, treating both wavelengths or $\mathrm{HbO}$ and $\mathrm{HbR}$ independently. Hence, some of these 2D analysis tools are forced to impose other non-physiological constraints, such as orthogonality or statistical independence. To overcome these limitations, a multidimensional $(\geq 3 \mathrm{D})$ approach called parallel factor analysis (PARAFAC) $)^{5,6}$, or less frequently referred to as canonical decomposition ${ }^{7}$, could be considered as an alternative for the analysis of fNIRS data.

Multidimensional decomposition was initially introduced in the field of psychometrics and linguistics as a tool for multi-factor analysis ${ }^{6-8}$. Over time, its use was extended to neuroimaging signals, such as the analysis of event-related potentials (ERPs) assessed by electroencephalography (EEG). For this application, decomposition took into account three dimensions: time, space and participants ${ }^{9,10}$. Later, PARAFAC was introduced for data analysis in continuous EEG recordings, taking into account the 3D structure of the time/frequency representation of the EEG signal (time $\times$ space $\times$ frequency $)^{11,12}$. The growing popularity of PARAFAC in neuroimaging stems from the intrinsic advantage of multidimensional decomposition, because most data gathered in neuroscience are naturally multidimensional. The data-driven approach could also enlighten new aspects of neuroimaging data ${ }^{13}$.

PARAFAC is a decomposition technique applicable to any dataset that can be described in more than two dimensions (e.g. time, space, frequency, participants, conditions, signal characteristics) and allows for the extraction of different signatures present in the data. It assumes multilinear relations between the different dimensions and usually does not need any other mathematical constraints to find a unique decomposition of the data. It may therefore be used in the data pre-processing steps to isolate artifacts, as well as in the actual data analyses to extract a predominant brain activation, or other relevant characteristics of the signal. Indeed, using a 3D wavelet transformation in EEG data, Miwakeichi and colleagues ${ }^{12}$ revealed PARAFAC as an appropriate tool for both the detection of ocular movement artifacts and the identification of dominant brain activation patterns. In their study, some of the components identified using PARAFAC were highly similar to those extracted with principal component analysis (PCA), a well-established approach for 2D decomposition. Specifically, significant overlap was shown for eigenvalues and peaks of time/frequency components, as well as their topographical representations found with PCA and PARAFAC. PARAFAC, using the dimensions of time, space and frequency, has also been applied successfully to the EEG data of individuals with epilepsy, for the purposes of artifact detection and the identification of aberrant cerebral activation ${ }^{14}$. Overall, these studies suggest that in the context of time $\times$ space $\times$ frequency analysis of EEG data, PARAFAC achieves comparable results to well-established approaches while allowing for easier interpretation, and doing so without imposing non-physiological constraints ${ }^{11}$. Multidimensional decomposition with PARAFAC is not limited to 3D and could potentially be applied to data with more dimensions. In the context of ERP data, PARAFAC as a five-way analysis has successfully been used to identify differences and common characteristics of inter-trial phase coherence across conditions and subjects, including the dimensions of time, channels, frequency, subjects, and conditions ${ }^{15}$.

Although PARAFAC has been applied on NIRS data in the food industry ${ }^{5}$, it has not yet been used in neuroscience. Its application to fNIRS brain data could allow simultaneous analysis of temporal, spatial and wavelength dimensions, the latter codifying the information for concentration changes in $\mathrm{HbO}$ and $\mathrm{HbR}$. Hence, after $\mathrm{HbO}$ and $\mathrm{HbR}$ concentration changes are derived from the optical intensities of both wavelengths, it can be referred to as the hemodynamic 
dimension. Thus, data analyses with PARAFAC could take into account all relevant dimensions contributing to the fNIRS signal, without artificially separating information delivered by the use of multiple wavelengths in each channel. As described above, because PARAFAC does not impose independence and orthogonality constraints ${ }^{12}$, it can represent the real underlying physiological signals that are mixed in a multilinear structure.

A highly useful application of PARAFAC is that of artifact correction. Although the fNIRS signal is considered to be relatively tolerant to movement ${ }^{16}$, it does include some movement artifacts that can lead to abrupt changes in the light intensity, thus reducing the quality of data. It has been shown that the dynamics of both wavelengths provide important information for artifact detection and correction ${ }^{17}$. However, current techniques for movement artifact correction typically do not take into account the information originating from both wavelengths, thus considering only two dimensions of the signal's structure simultaneously (e.g. time and space) ${ }^{2-4}$.

The bi-dimensional (time-space) decomposition of fNIRS data into components for the purposes of artifact correction is typically performed using principal component analysis (PCA) and independent component analysis (ICA). Although there are several ways to approach PCA (e.g. dimensionality reduction ${ }^{18}$, classification ${ }^{19}$ ), from the signal decomposition point of view, PCA aims at extracting the principal component, that is the component that explains the greatest amount of variance of the signal ${ }^{20}$. It has been used for artifact removal because it helps to isolate the artifact's signature, or to extract other relevant activities in $\operatorname{ANIRS}^{3,4,20,21}$. The data is thus decomposed into a sum of components, each one formed by the product of two vectors: one representing the temporal principal component and the other, the corresponding topography (scores for each channel). A basic problem with PCA is that the components defined by only two signatures (time and space) are not uniquely determined. Therefore, in PCA, orthogonality is imposed between the corresponding temporal signatures of the different components ${ }^{4,12,22}$. Orthogonality is, however, a rather non-physiological constraint. Even with this restriction, the extracted principal components are not completely unique, given that the arbitrary rotation of axes does not change the explained variance of the data. This has led researchers to use different mathematical criteria as the basis for choosing specific rotations (e.g. Varimax, Quartimax, Promax). In fNIRS, PCA has also been applied to target time intervals (tPCA), that is to say only during periods where artifacts related to articulation or other head movements occurred, instead of during the entire unsegmented signal ${ }^{23,24}$. This type of targeted correction resulted in better signal quality, as compared to wavelet-based filtering and spline interpolation, while also reducing the risk of altering the signal's global integrity ${ }^{24}$.

More recently, ICA has become another popular tool for data decomposition in $\mathrm{fNIRS}^{25-}$ 27. It has the benefit of preventing rotational freedom ${ }^{28}$. However, uniqueness is achieved at the cost of imposing a constraint even stronger than orthogonality, namely, statistical independence of the temporal signatures ${ }^{29,30}$. Statistical independence is appropriate for identifying artifacts with spatio-temporal signatures that are very different to those of neural activity (e.g. ocular movements), but is less appropriate for artifacts that share spatio-temporal characteristics with neural signals. What is more, ICA is commonly applied to the entire signal in contrast to a target decomposition as introduced in the previous paragraph for $\mathrm{PCA}^{27}$. It may thus be more challenging to achieve a satisfying correction of irregular movement artifacts. In ICA, the maximal number of components (hypothesized sources) equals the number of observations, which in neuroimaging is often rather high. It could therefore, become difficult to identify the artifact's signature, which could be split into several components. 
In addition to being used for movement artifact correction, PARAFAC could also be considered as a tool for the analysis of the hemodynamic response. Typically, cerebral activation is characterized by a relative increase of the $\mathrm{HbO}$ concentration level, with a slight decrease of the $\mathrm{HbR}$ concentration level ${ }^{31-34}$. Despite the advantage of measuring signals that reflect both $\mathrm{HbO}$ and $\mathrm{HbR}$ concentration changes, the estimation of cerebral activation in fNIRS studies is often mainly based on $\mathrm{HbO}$ concentration changes ${ }^{33,35}$. This is because the $\mathrm{HbO}$ increase is often easier to identify than changes to $\mathrm{HbR}$ concentration, due to its greater amplitude and the higher signalto-noise ratio. Furthermore, contradictory findings exist regarding the occurrence of HbR decrease, which is also why many studies favor the interpretation of $\mathrm{HbO}$ concentration changes only, without further investigation of $\mathrm{HbR}$ changes. However, ignoring concentration changes in $\mathrm{HbR}$ poses the risk of false positive results, for instance because higher levels of $\mathrm{HbO}$ could actually, for example, be due to an artifact ${ }^{17}$. Simultaneous treatment of both wavelengths or $\mathrm{HbO}$ and $\mathrm{HbR}$ with PARAFAC would help to overcome this limitation and allow for the identification of typical hemodynamic signatures, i.e. increase of $\mathrm{HbO}$ and slight decrease of $\mathrm{HbR}$.

The current study aims to validate PARAFAC for the decomposition of the fNIRS signal to (1) extract and correct movement artifacts, and (2) characterize task-related brain activation. For that purpose, we acquired fNIRS data of 18 healthy adults while they performed aloud a semantic verbal fluency task. First, we compared the use of PARAFAC to two commonly used decomposition techniques for motion artifact correction (tPCA and ICA). Subsequently, we evaluated the spatial and temporal characteristics of language-related brain activation as identified with the PARAFAC model, and compared them to those revealed by commonly used approaches (general linear model, global average).

\section{Methods}

\section{$2.1 \quad$ Sample}

Eighteen native French-speaking adults $\left(\mathrm{M}_{\mathrm{age}}=22.8\right.$ years, $\mathrm{SD}=2.0$ years; female $=10$ / male $=8$ ) participated in this study. Inclusion criteria were: being French unilingual (no second language acquired before the age of five), right-handed, born at term, having normal hearing, normal or corrected-to-normal vision, and no history of neurological, chronic or psychiatric disorders. Experimental procedures were approved by the local ethics committee.

\subsection{Procedure and verbal fluency task}

Participants sat comfortably in a soundproof and electrically shielded room. They were instructed to relax, to avoid any intentional movements or muscular tension, and to fix their gaze on the center of a screen placed at a distance of $114 \mathrm{~cm}$. Participants underwent a fNIRS recording while they performed a verbal fluency task previously validated for expressive language-related activation $^{36-38}$. The task consisted of 11 different semantic categories (e.g. animals, colors, fruits, etc.), for which participants had to say aloud as many words as possible for each category. The name of the category was presented visually on the screen (Presentations ${ }^{\circledR}$, Neurobehavioral Systems, 2018). We used a block design paradigm in which periods of rest (fixation cross presented on the screen) and task (semantic category) alternated in 30-seconds intervals. A video camera filmed the entire session, allowing for the visual identification of movements during offline preprocessing. Audio data was used to count the number of words reported per category and to identify the timing of the words, since articulation can induce movement artifacts. 


\section{3 fNIRS data acquisition}

fNIRS data was acquired using a frequency-domain fNIRS device (Imagent Tissue Oxymeter, ISS Inc., IL, USA) equipped with 60 emitters, each regrouping two light sources of different wavelengths $(690$ and $830 \mathrm{~nm}$ ), and 14 detectors. Emitters and detectors coupled at a distance of 3-4.5 cm allowed for the recording of 106 channels for both wavelengths. Optodes were held in place perpendicularly, using a cap that was fitted on the head of participants in accordance with the 10-20 system ${ }^{39}$. Optical intensity was measured with a sampling rate of 19.53 $\mathrm{Hz}$ (Boxy, ISS Inc., IL, USA). The setup covered both hemispheres equally and included the regions of interest for the investigation of language functions, i.e. frontal, temporal and parietal lobes (see Fig. 1). A short-distance emitter-detector pair with a distance of $1 \mathrm{~cm}$ was placed at the midline in the occipital region. This short-distance pair enables the control for continuous physiological contamination (cardiac frequency, respiration, Mayer waves) in the superficial layer of the head, which is not directly related to cerebral activation ${ }^{40,41}$.
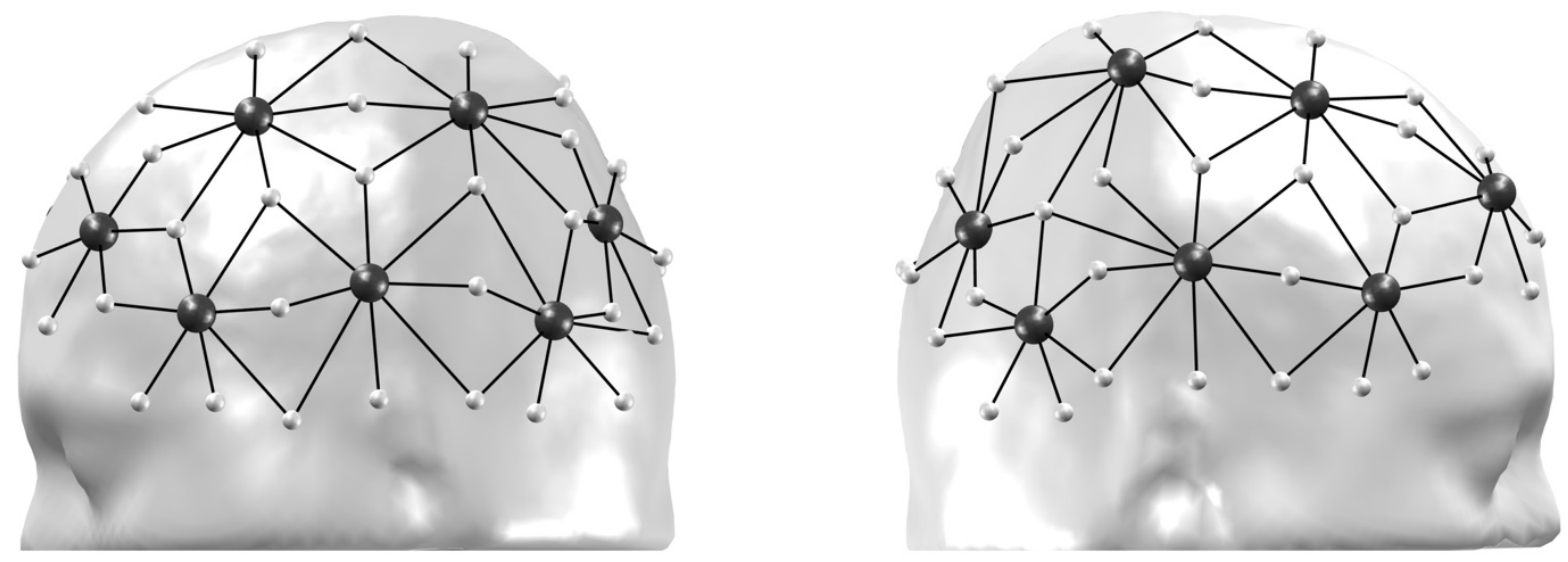

Fig. 1 fNIRS setup with 14 detectors (large dark circles), and 60 light emitters (small light dots) each sending light at two different wavelengths $(\lambda=690$ and $830 \mathrm{~nm}$ ): The reflection of light emitted by each source is captured by one or several proximal detectors. The combination of a light emitter and a detector represents a channel (dark grey lines), for a total of 106 channels.

\section{$2.4 \quad$ Pre-processing}

Data analysis was conducted with the use of a homemade toolbox (LIONNIRS) adapted in SPM12 (Statistical Parametric Mapping) ${ }^{42}$ in MATLAB® (The MathWorks, Inc., MA, USA). Preprocessing steps included segmentation of the signal into blocks of 50 seconds $(5 \mathrm{~s}$ resting-state baseline, $30 \mathrm{~s}$ task and $15 \mathrm{~s}$ resting-state). Every block was normalized by dividing each time point by the mean of the baseline. A low-pass filter with a cut-off frequency of $0.2 \mathrm{~Hz}$ was applied, in order to remove oscillations caused by heartbeat and respiration.

\subsection{Movement artifact detection and correction}

Since our language paradigm required participants to name words aloud, artifacts were mostly due to facial movements related to the muscular contraction of articulation. We performed 
a semi-automatic artifact detection, using primarily an algorithm to automatically mark segments where an abrupt change exceeded three times the average variance of the signal ${ }^{43}$. Visual interrater inspection based on the signal's characteristics and the video recording led to the final identification of the time intervals with artifacts. These time intervals would subsequently be used to compare the efficacy of different movement artifact correction techniques intended to increase the signal quality (see Sec. 4.7 on statistical analysis). Artifact correction was then applied to these segments using PARAFAC and two commonly used 2D decomposition techniques in fNIRS, namely those of tPCA and ICA.

\subsubsection{Two-dimensional signal decomposition for artifact detection and correction}

For both ICA and tPCA, the decomposition of the 2D data matrix $X$ (whose elements $x_{i j}$ are indexed by $i$ channel and $j$ time points), leads to $N_{f}$ components as defined in Eq. 1 and illustrated in Fig. 2a:

$$
x_{i j}=\sum_{f=1}^{N f} a_{i f} b_{j f}+\varepsilon_{i j}
$$

The matrix combines two separate data matrices $\left(X_{1}, X_{2}\right)$ corresponding to the $2 \mathrm{D}$ structure for both wavelengths. The maximum number of components $N_{f}$ could be equal to or less than the smaller dimension of the $X$ matrix dimension, i.e. the number of channels or number of time points. Each component is modeled as the product of two factors/vectors which represent signatures of the space $\left(a_{f}\right)$ and time $\left(b_{f}\right)$ dimensions. The unexplained part of the data is considered irrelevant activity or noise $(\varepsilon)$.

Artifact correction with tPCA was performed on each time interval previously identified as containing artifacts. From the obtained components we subtracted only the first, which explains most of the variance based on the assumption that the highest variance in the data for each segment is assumed to be caused by the artifact. Artifact correction with ICA was done using Brain Vision Analyzer (Brain Products GmbH, Gilching, Germany), and followed the steps proposed by Plank $^{44}$. First, raw fNIRS data were exported and ICA was applied on the entire unsegmented signal, as for the method commonly reported in the literature ${ }^{27}$. Noisy intervals reflecting artifacts were identified by semi-automatic inter-rater artifact detection (see Sec. 4.5). We visually selected the ICA components reflecting the artifact, based first on their time course, i.e. high variation of light intensity similar to the artifact signature. Secondly, we rejected those whose subtraction would induce artificial artifacts elsewhere. Data was subsequently imported back into the LIONNIRS toolbox in order to apply segmentation and normalization (see Sec. 4.4).

\subsubsection{Multidimensional signal decomposition}

The fNIRS data naturally offers the time courses of all channels for two wavelengths, i.e. two separate data matrices. This data can be arranged in a tri-dimensional array, as illustrated in Fig. 2b. The dimensions of this 3D array are time (indexed by the time points in the analyzed segment), space (indexed by 106 channels) and wavelength (indexed by the two wavelengths). As explained above, PARAFAC establishes a trilinear decomposition of each element of the fNIRS data array $\left(X_{t s w}\right)$ in $N_{f}$ components, each being the product of three factors ${ }^{5}$ :

$$
X_{t s w}=\sum_{f=1}^{N f} a_{t f} b_{s f} c_{w f}+\varepsilon_{t s w}
$$


The estimated factors, $a_{t f}, b_{s f}$ and $c_{w f}$ are the elements of the so-called loading matrices $A, B$ and $C$, whose column vectors $a_{f}=\left\{a_{t f}\right\}, b_{f}=\left\{b_{s f}\right\}, c_{f}=\left\{c_{w f}\right\}$, will represent the temporal, spatial and wavelength signatures of each component (Fig. 2b). The main advantage of this method is that it provides a unique decomposition of the fNIRS data into components reflecting different activities that do not need to be orthogonal or statistically independent in any of the dimensions. As long as an activation shows a different behavior in one of the dimensions, it can be extracted as a separate component. In this application, the spatial dependency between wavelengths is therefore exploited in order to perform the decomposition.

The uniqueness of the solution is guaranteed when the number of components $\left(N_{f}\right)$ is smaller than the sum of the ranks of the three loading matrices. In the case of noisy data, it is very likely that the loading matrices are always full rank. Uniqueness is thus guaranteed as long as the number of components $\left(N_{f}\right)$ is smaller than half the sum of the number of time points $\left(N_{t}\right)$, number of channels $\left(N_{c}\right)$, and number of wavelengths $\left.\left(N_{w}\right): N f \leq \frac{\left(N_{t}+N_{c}+N_{w}\right)}{2}-1\right)$. For instance, even in a small array of 20 time points and eight channels at two different wavelengths, a unique decomposition could be achieved, using up to 14 components $(20(t)+8(s)+2(w)=30 \div 2=$ $15-1=14(N f)$ ). Computationally, the decomposition is achieved by the Alternating Least Squares algorithm, as it has been used in previous studies on neuroscience data ${ }^{11,12,14,15}$. The only (trivial) indeterminacies in the least square solution are the order of the components and the relative scaling of the signatures. 
A

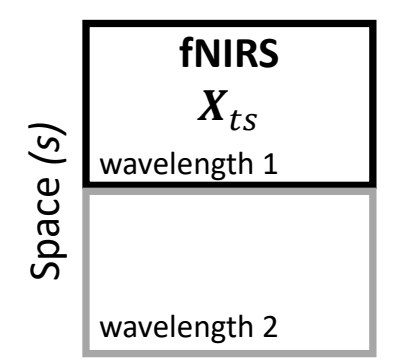

Time $(t)$

B

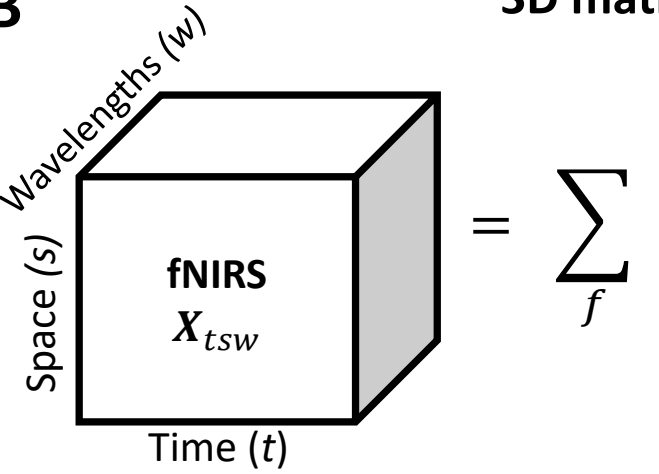

2D matrix decomposition

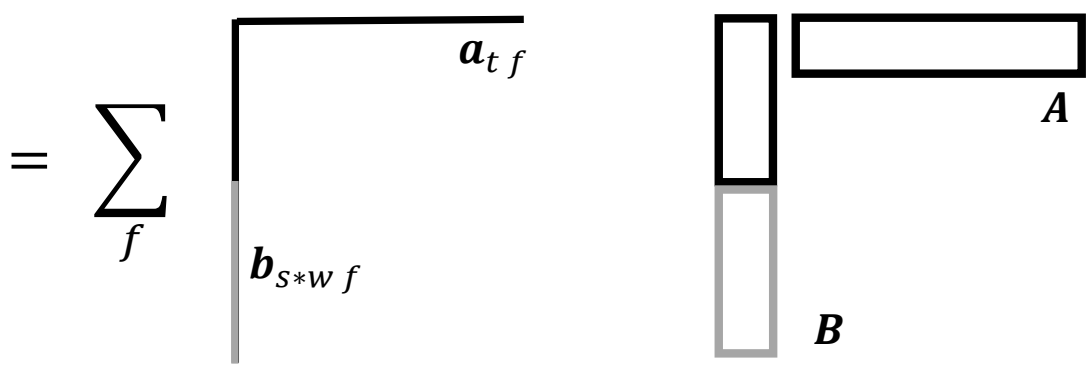

Fig. 2 Schematic representation of the decomposition models applied to fNIRS data: (a) The data $X$ is arranged as a $2 \mathrm{D}$ data array by vertically concatenating the 2D matrices with dimensions being space $(s)$ and time $(t)$ for each wavelength $(w)$. tPCA/ICA decomposes the array into components, each being a bilinear product of the loading vectors representing temporal $\left(a_{t f}\right)$ and spatial signatures $\left(b_{s * w}\right)$. The latter is formed by the spatial signatures for the different wavelengths which are represented in components without taking into account their spatial dependence, i.e. for the same temporal signature of each component, there will be two topographies corresponding to the two wavelengths. Matrices $A=\left\{a_{f}\right\}$ and $B=\left\{b_{f}\right\}$, contain as columns the temporal and spatial signatures for all components, respectively. (b) The data $X$ is arranged as a 3D data array with dimensions being time $(t)$, space $(s)$ and wavelengths $(w)$. PARAFAC decomposes this array into the sum of components, each being a trilinear product of loading vectors representing temporal $\left(a_{t f}\right)$, spatial (channel, $\left.b_{s f}\right)$ and spectral (wavelength, $c_{w f}$ ) signatures. In practice, the decomposition consists of finding the matrices $A=\left\{a_{f}\right\}, B=\left\{b_{f}\right\}$ and $C=\left\{c_{f}\right\}$ that explain $X$ with minimal residual error.

In this work, we used the PARAFAC implementation from the N-way Toolbox ${ }^{45}$, which was included into the LIONNIRS toolbox so as to visualize and apply the decomposition exclusively during selected time intervals and channels. Components were ordered according to their importance in explaining the data's variance (similar to PCA). The scale of the data was kept in the temporal signatures, while the other dimensions are normalized so as to have Frobenius norms equal to one.

Since measures from both wavelengths are sampled simultaneously at each specific position on the scalp, movement artifacts affect their amplitudes similarly. During time intervals containing artifacts, the time courses of the signal from the two wavelengths commonly show a drastic and correlated increase as compared to the task-related or baseline signa $1^{17}$. By using multidimensional PARAFAC decomposition, we can take advantage of this information for the 
adequate selection of components of the artifact's signature. PARAFAC decomposition was specified to extract between two to four components, allowing a clear separation of the artifact signatures representing the artifacts characteristics from the rest of the signal. The appropriate components were selected based on (1) a visual inspection of their temporal overlap with the artifact, (2) the smallest number of possible components that would sufficiently correct the artifact, and (3) components showing similar weights for both wavelengths. Usually, one to three components were needed in order to achieve a satisfactory correction of the artifact, without removing the characteristics related to the true cerebral activity.

\subsection{Characterizing $f$ NIRS activation patterns}

As a second part of the study, we applied PARAFAC and other commonly used methods to the clean signal to characterize activation patterns and assess the utility of PARAFAC to model the brain response in fNIRS. First, the optical intensity of the two wavelengths was transformed into relative concentration changes of $\mathrm{HbO}$ and $\mathrm{HbR}$, done so by using the modified Beer-Lambert $\mathrm{Law}^{46}$. For each participant, all blocks underwent detrending in order to align the beginning and end of the segments. Then, in order to characterize the cerebral activation pattern related to the verbal fluency task, three different approaches were applied to the 30-s task period of each block: (i) the General linear model (GLM), (ii) the global averaged concentration changes of each channel, and (iii) the PARAFAC analysis.

(i) General linear model (GLM) assumes that there exist linear relations between different inputs ${ }^{47}$. It has been applied in several fNIRS studies to specify the hemodynamic response function ${ }^{4,48,49}$. For each participant, the task-related cerebral activation $(y)$ is modeled separately for every single block, using a GLM defined as

$$
y=\beta_{0}+\beta_{1} a+\beta_{2} b+\varepsilon
$$

In this equation, $\beta_{0}$ is a constant. $\beta_{1}$ represents the relative contribution of the modeled hemodynamic response ${ }^{50}$ (concentration changes of $\mathrm{HbO}$ and $\mathrm{HbR}$ respectively) based on the temporal characteristics of the stimuli defined in $a . \aleph_{2}$ represents the contribution of physiological components measured with the short-distance $(<1 \mathrm{~cm})$ source-detector pair $b$, and $\varepsilon$ represents the unexplained part of the data related to noise or other unknown sources. On a subject-level, individual $\beta_{1}$ of each channel were averaged. Subsequently, the obtained $\beta$-values were subjected to group-level analysis using a one sample t-test against 0 to identify channels showing significant temporal overlap with the hemodynamic response function, which was modeled based on the stimuli presentation ${ }^{51-53}$. A critical $p$-value of 0.05 after Bonferroni correction for multiple comparisons was interpreted as a significant activation. This allowed to derive the temporal and spatial characteristics of the dominant cerebral activation pattern during the semantic verbal fluency task, as revealed by the GLM.

(ii) The computation of the global averaged concentration changes of each channel is a straightforward approach to explore the spatial distribution of the task-related hemodynamic response ${ }^{4,20}$. The relative weight of the short-distance channel $\left(\beta_{2}\right)$ allows for the subtraction of physiological components from the fNIRS signal. The global average of $\mathrm{HbO}$ and $\mathrm{HbR}$ concentration changes, for every channel and across each block, was calculated on the corrected signal. For each block, the time points covering from 5 to 20 seconds after task onset were averaged. Channels with significant concentration changes were identified using t-tests on each channel's average signal as compared to 0 . 
(iii) A PARAFAC decomposition with a single component was applied for each block of each participant's data set after the subtraction of physiological noise from the short-distance channel $\left(\beta_{2}\right)$. We assumed that after removing artifacts and physiological noise, the activation related to the verbal fluency task would stand out as the main component of the PARAFAC decomposition. In some cases, we obtained signatures with an "inverted" sign (i.e. showing a decrease in concentration changes of $\mathrm{HbO}$ related to the task instead of an increase, with the inverse phenomenon for $\mathrm{HbR}$ ) due to the indeterminacy of the scale of the signatures (see Fig. 6 in Appendix for a detailed description of the different cases found). In these particular cases, the signatures obtained by PARAFAC were subjected to a sign adjustment ${ }^{5}$. We based ourselves on the experimental fact that $\mathrm{HbO}$ concentration changes are typically higher than those for HbR. Then, the sign of each pair of signatures was changed to obtain components that represented activation scores of $\mathrm{HbO}$ higher than those of $\mathrm{HbR}$, while the mean of the temporal signature remains positive. This type of empirical adjustments has also been reported in other neuroimaging studies $^{15,54}$.

Similar to the coefficient in the GLM, which indicates how much the signal of a specific channel contributes to the modeled hemodynamic response based on the time course of the stimuli, the temporal signature of the PARAFAC component should represent the time course of the cerebral activation related to the task. The spatial signature (topography) shows how much each channel contributes to the selected component, while the hemodynamic signature contains two relative scores specifying how much its activation is represented in the $\mathrm{HbO}$ and $\mathrm{HbR}$ signals.

\subsection{Statistical analysis}

In order to evaluate the use of PARAFAC for the correction of movement artifacts in fNIRS data, its performance was compared to the well-established 2D decomposition techniques (tPCA and ICA), with the use of two quality measures. First, for each data segment with an artifact we calculated an estimation of the signal-to-noise ratio (SNR) before and after artifact correction, as defined by Sweeney and colleagues ${ }^{55}$ :

$$
S N R=10 \log _{10} \frac{\sigma_{x}^{2}}{\sigma_{e}^{2}}
$$

Here, $\sigma_{x}^{2}$ represents the signal's variance computed in segments of data, where no artifacts were identified, while $\sigma_{e}^{2}$ represents the variance of the analyzed signal in a segment where an artifact was identified. As the latter will usually be higher than the former (since it comprises both the variance of the physiological signal and the variance of the artifact), the SNR was expected to be negative whenever an artifact is present in the analyzed segment. After removing the artifact from the analyzed signal, the SNR was computed in order to identify how effectively the artifact was removed from the signal. A zero value for the SNR would indicate equal variances, meaning that an artifact was eliminated to the extent that the corrected signal did not differ from periods in which no artifact was identified. A negative value would suggest that there was some remaining variance from the artifact, and a positive value would suggest that elimination of the artifact probably subtracted part of the relevant physiological activity. Repeated-measures analysis of variance (ANOVA) and post-hoc tests with Bonferroni corrected p-values were used to compare statistically the differences between the SNR of the uncorrected signal and the signal corrected with the three different methods for artifact correction. 
Pearson's correlation coefficients between the time courses for both wavelengths were used as a second measure of the efficiency of the artifact correction ${ }^{17}$. This was based on the assumption that a high correlation coefficient indicates the presence of artifactual signals, since in a clean signal these temporal courses appear much less correlated. We used Fisher's transformation to convert correlation coefficients into z-values to obtain an approximately normal distribution. Repeated-measures ANOVA allowed for the comparison of the mean Pearson's correlation coefficients between the uncorrected segments to those corrected, and this for each of the three methods as well as for a time interval without artifacts (5-s baseline at rest). Pearson's correlation of the 5-s baseline served as a reference for a segment without artifacts and with no task-related cerebral activation.

Regarding brain activation related to the verbal fluency task, we aimed to compare temporal (time), topographical (space), and hemodynamic $(\mathrm{HbO} / \mathrm{HbR}$ ) results specified by the PARAFAC component with results from the hemodynamic response function (HRF) estimated with the GLM, as well as the global average for each channel. Temporal and hemodynamic characteristics were visually compared, based on time courses of the task-related concentration changes in $\mathrm{HbO}$ and $\mathrm{HbR}$ within the regions of interest (ROIs) as revealed by each of the three methods (PARAFAC, GLM, global average). Our first ROI was defined as the left Broca's area (according to Brodmann 44, 45) and was identified by choosing the two channels showing maximum task-related activation in the left inferior frontal lobe for all participants. Our second ROI was defined as the right Broca's area, thus including the two homologous channels in the right hemisphere (see Fig. 4). Comparisons of the spatial characteristics of the task-related activation were conducted for the mean concentration changes of every channel. A one-sample t-test between the relative weight (PARAFAC), the $\beta_{1}(\mathrm{GLM})$ and the mean $\mathrm{HbO}$ and $\mathrm{HbR}$ concentration changes (global average) were tested against 0 .

\section{Results}

From the whole sample of 18 participants, one participant was excluded from analyses, as artifact detection revealed a signal containing too many segments with noise, thus precluding the use of the quality measures to compare artifact correction techniques. The final sample for the validation of using PARAFAC for artifact correction included 17 participants who, on average, completed $10.4 \pm 0.9$ blocks of the verbal fluency task. During each 30 -s block of the verbal fluency task, participants named an average of $14 \pm 2.2$ words.

\subsection{Artifact correction}

Our comparison of the signal-to-noise ratio (SNR) included 1,401 time intervals with previously identified artifacts, and which contained a total number of 19,630 channels for $\mathrm{HbO} / \mathrm{HbR}$. The uncorrected signal with artifacts has a mean SNR value of $M=-0.69 \mathrm{~dB}(S D=$ $0.65 \mathrm{~dB})$; the signal corrected with PARAFAC, $M=-0.31 \mathrm{~dB}(S D=0.58 \mathrm{~dB})$; tPCA, $M=-0.33$ $\mathrm{dB}(S D=0.58 \mathrm{~dB})$; and ICA, $M=-0.50 \mathrm{~dB}(S D=0.64 \mathrm{~dB})$ respectively. Repeated-measures ANOVA for all conditions (signal without correction, signal corrected with PARAFAC, tPCA or ICA) revealed a significant global effect, $F(2.3,44966.3)=7550.7, p<0.001, n=0.28$. Pairwise comparisons with Bonferroni correction indicated a significant difference between all SNR. All three artifact correction methods lead to signals with an increased SNR, thus suggesting that all of them resulted in a significant improvement of the signal's quality, as compared to the uncorrected 
signal (see Table 1). According to the mean SNR, PARAFAC achieved the highest correction, followed by tPCA and then ICA. As the number of segments used for statistical analyses was rather large, it was relevant to consider effect sizes. The differences between the SNR of the PARAFAC corrected signal and the uncorrected signal, as well as between PARAFAC and ICA corrected signal, represented large effects $\left(n=0.45\right.$ and $n=0.13$, respectively $\left.{ }^{56}\right)$. In contrast, the difference in SNR between TPCA and PARAFAC corrections was a rather small effect $(n=0.002)$.

Table 1 Pairwise comparisons of the signal-to-noise ratio of the signal after using the different correction techniques and the original uncorrected signal.

\begin{tabular}{lcccc}
\hline & Mean Signal-to-noise ratio in dB (SD) & PARAFAC & tPCA & ICA \\
\hline Uncorrected & $-0.69 \pm 0.65$ & $-0.38^{*}$ & $-0.37^{*}$ & $-0.19^{*}$ \\
PARAFAC & $-0.31 \pm 0.58$ & & $0.01^{*}$ & $0.19^{*}$ \\
tPCA & $-0.33 \pm 0.57$ & & & $-0.18^{*}$ \\
ICA & $-0.50 \pm 0.64$ & & & \\
\hline
\end{tabular}

Note. Parallel factor analysis (PARAFAC); target principal component analysis (tPCA); independent component analysis (ICA). The table contains the mean differences between the signal-to-noise ratio of the data interval with artifacts for the uncorrected signal, and the signal where the three different artifact correction techniques, PARAFAC, tPCA and ICA, were applied.

*Significant difference at $p<0.001$ after Bonferroni correction for multiple comparisons.

Within segments with artifacts $(n=9815)$, Pearson's correlations between the time courses of the signals for both wavelengths were computed for all channels (see Table 2). Mean correlation coefficients across all these segments were compared for the uncorrected signals, the signals corrected with each of the three different artifact correction techniques, and the artifact-free signals corresponding to a baseline period. During the baseline, the time courses of the different wavelengths correlated on average at $r=0.47(\mathrm{SD}=0.33)$. Both wavelengths correlated on average at $r=0.74(\mathrm{SD}=0.29)$ for the uncorrected segments, at $r=0.51(\mathrm{SD}=0.38)$ after PARAFAC correction, at $r=0.53(\mathrm{SD}=0.38)$ after tPCA correction, and at $r=0.67(\mathrm{SD}=0.38)$ after ICA correction. The uncorrected signal with artifacts corresponded to the highest mean correlation, followed by ICA, tPCA, PARAFAC, and finally, the baseline reference.

Mean correlation coefficients of all channels were transformed into z-scores using Fisher transformation, in order to achieve an approximately normal distribution. Repeated-measures ANOVA revealed a significant difference between the five conditions: $F(3.2,30969.8)=1908.8$, $p<0.01, n=0.16$. Pairwise comparisons with Bonferroni corrected p-value for multiple comparisons further indicate significant differences of Pearson's correlation of the time course of both wavelengths between PARAFAC and all other conditions (see Table 2): 1) PARAFAC vs. uncorrected signal: $p<0.001, n=0.35$; 2) PARAFAC vs. tPCA: $p<0.001, n=0.01$; 3) PARAFAC vs. ICA: $p<0.001, n=0.14$; and 4) PARAFAC vs. baseline: $p<0.001, n=0.01$. Differences in Pearson's correlation coefficients between the PARAFAC corrected signal and the uncorrected signal $(n=0.35)$, as well as between PARAFAC and ICA corrected signal $(n=0.18)$, represented large effects ${ }^{56}$. Differences in correlation coefficients between PARAFAC and $\operatorname{tPCA}(n=0.02)$, as well as between PARAFAC and the baseline signal $(n=0.02)$, represented small to medium effects. 
Table 2 Mean Pearson's Correlations between signals measured with two different wavelengths.

\begin{tabular}{|c|c|c|c|c|c|}
\hline & $\begin{array}{l}\text { Mean Pearson's } \\
\text { correlation }( \pm \mathrm{SD})\end{array}$ & PARAFAC & tPCA & ICA & Baseline \\
\hline Uncorrected & $0.74 \pm 0.29$ & $0.228^{*}$ & $0.203 *$ & $0.063 *$ & $0.272^{*}$ \\
\hline PARAFAC & $0.51 \pm 0.38$ & & $0.025^{*}$ & $0.165^{*}$ & $0.044^{*}$ \\
\hline tPCA & $0.53 \pm 0.38$ & & & $0.150^{*}$ & $0.069^{*}$ \\
\hline ICA & $0.67 \pm 0.38$ & & & & $0.209^{*}$ \\
\hline Baseline & $0.47 \pm 0.33$ & & & & \\
\hline
\end{tabular}

Note. The table shows the mean differences between the Pearson's correlations obtained for the data segments with artifacts (uncorrected signal), data segments corrected by the three artifact correction techniques, PARAFAC, tPCA and ICA, and also for the baseline segments at rest, where no artifacts were identified.

*Significant effects with Bonferroni corrected p-value for multiple comparisons: $p \leq 0.001$.

Figure 3 provides an example of target artifact correction with PARAFAC and TPCA, illustrating the difference between the $3 \mathrm{D}$ and $2 \mathrm{D}$ target decomposition for motion artifact correction, respectively. The time course of the three components obtained with PARAFAC decomposition allowed to easily differentiate between the two components with distinct artifact signatures, and the one component representing the clean signal (Fig. 3, left box). Spatial distribution of each component is shown as a loading matrix and helped to identify in which channels (localization) the artifact's signatures were most important (Fig. 3, upper middle box). The two components that appear to represent the artifact showed similar scores for both wavelengths, as represented in the wavelength signatures (Fig. 3, upper middle box). Artifacts are known to affect both wavelengths similarly, thus equal contribution to a component of both wavelengths is considered a reliable characteristic of an artifact component ${ }^{17}$. On the one hand, signal correction that removes the two PARAFAC components subsequently resulted in a less noisy time interval (Fig. 3, right box). On the other hand, the first principal component of tPCA seems to catch the characteristics of one of the two distinct artifacts, but not both. Therefore, tPCA only seems to achieve a partial correction. 

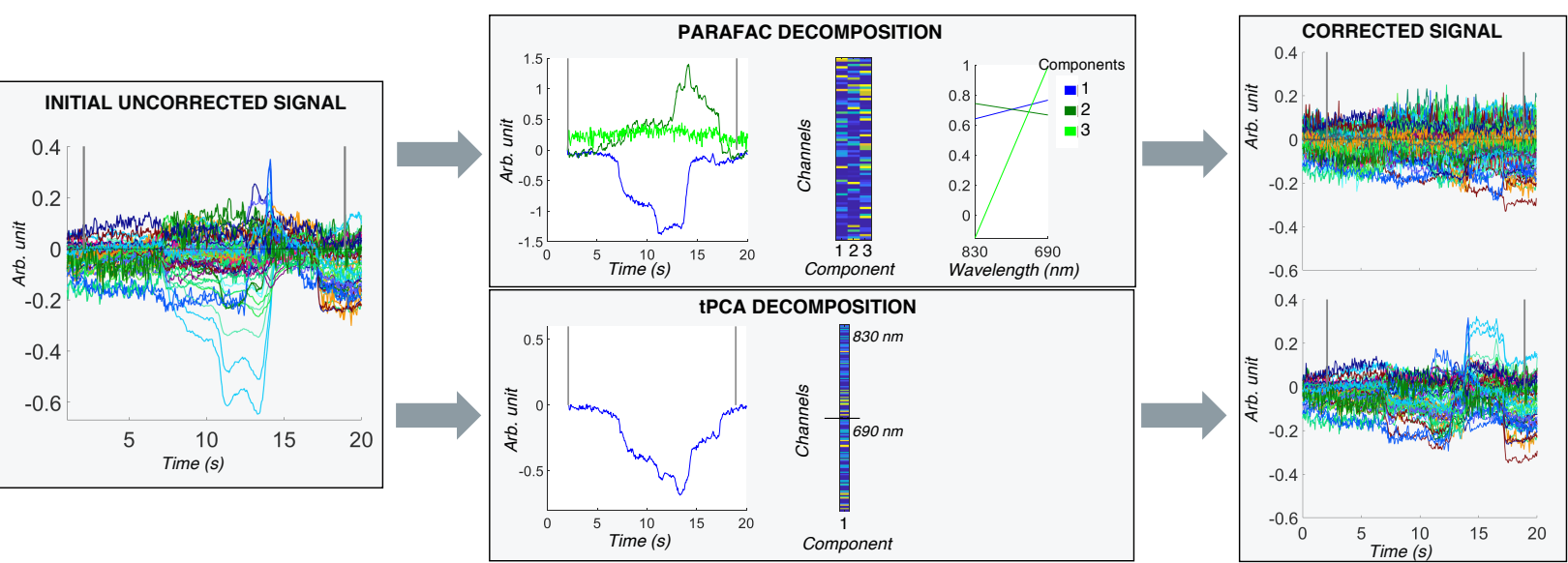

Fig. 3 Example of target 3D PARAFAC and 2D tPCA decompositions to correct motion artifacts in a fNIRS data set: The left box shows the initial (uncorrected) data segment. The upper box in the middle contains the temporal, spatial (channel) and wavelength signatures of the components identified with PARAFAC, respectively. The lower box in the middle includes the temporal and spatial characteristics of the 2D signal, as revealed by the first principal component of tPCA. The right box reveals the corrected signal, illustrating the efficacy of movement artifact correction with PARAFAC in upper part and IPCA in the lower part, respectively.

\subsection{Characterization of task-related cerebral activation}

Four participants were excluded for the characterization of brain activation, for a total of 14 participants included in the analyses. In addition to the one excluded for the first part of the study (artifact correction), three participants did not achieve satisfactory data quality even after artifact correction (i.e. too many channels were missing due to aberrant amplitude or frequency). The complete topographical representation of all significant cerebral activations for the three approaches, PARAFAC, GLM, and global average, are illustrated in Fig. 4. Results revealed significant activations for all three approaches in our ROIs, the left Broca's area and its homologous region (see Table 3). Figure 5 illustrates the temporal courses of the task-related cerebral activation obtained from the PARAFAC component, GLM and global averaging within our ROIs during the 30 -s period of the verbal fluency task. 


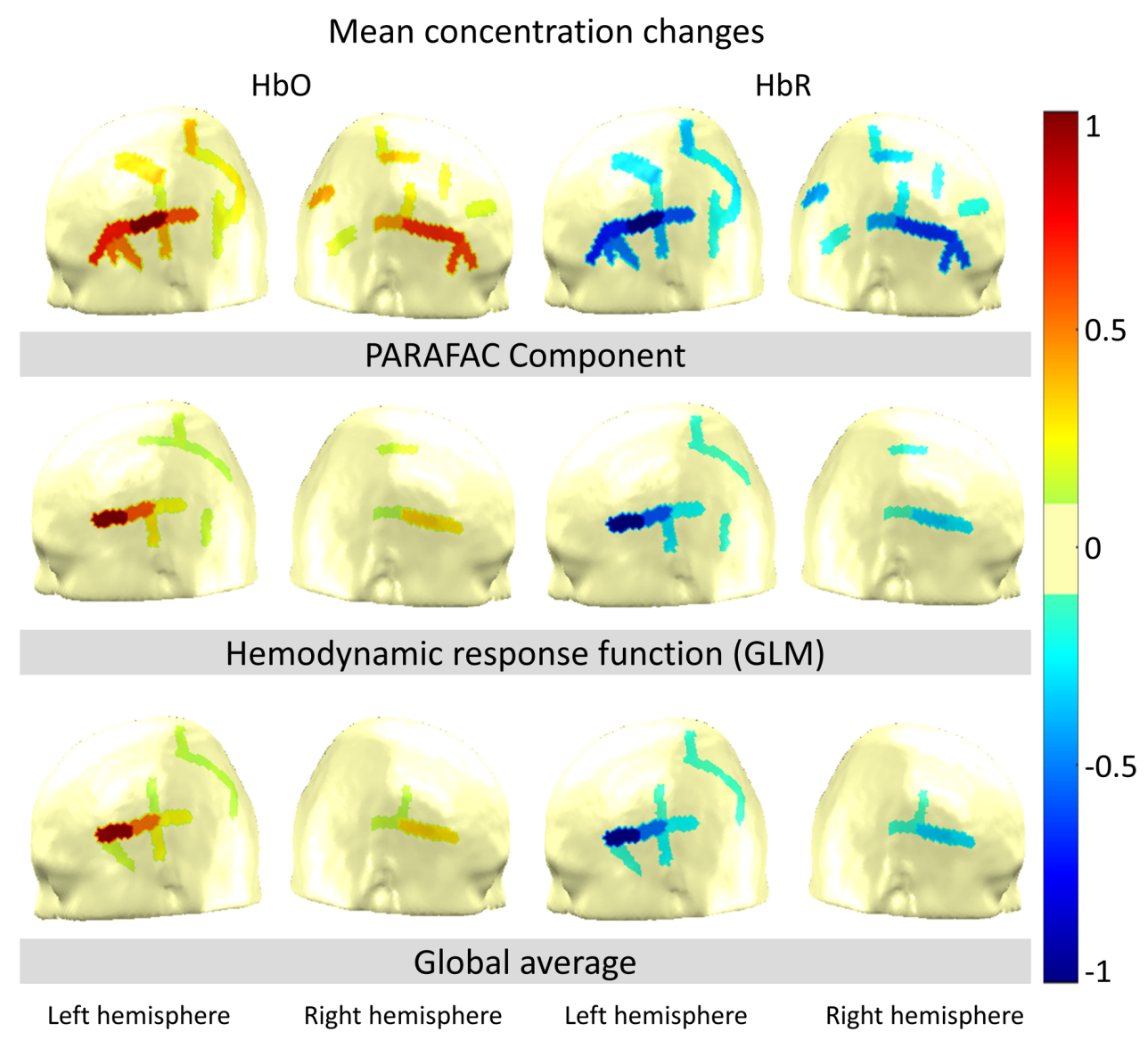

Fig. 4. Topographical representation of significant task-related cerebral activation: Cerebral activation during the 30$\mathrm{s}$ verbal fluency task as revealed by (top row) the PARAFAC component (i.e. time dimension $\times$ hemodynamic dimension), (middle row) the HRF from the GLM, and (bottom row) the global average. Only significant mean concentration changes in $\mathrm{HbO}$ (left) and $\mathrm{HbR}$ (right), as revealed by the one-sample t-tests against 0 , are illustrated $(t$ $\geq 1.64$ for $\mathrm{HbO}, t \leq-1.64$ for $\mathrm{HbR}$; one-tailed $p<.05$ ). Relative contribution of each method (i.e. mean relative weights for the PARAFAC component, mean Beta values for the HRF specified in the GLM, and global average per channel) are displayed in a relative scale ranging from -1 to 1 . A value of 1 indicates maximum increase of $\mathrm{HbO}$ concentration, a value of -1 represents a maximum decrease of $\mathrm{HbR}$ concentration. 
Table 3 Significant task-related cerebral activation associated to the verbal fluency task as revealed with PARAFAC, GLM and global average for left and right Broca's areas.

\begin{tabular}{ccccccc}
\hline \multicolumn{2}{c}{ PARAFAC } & \multicolumn{2}{c}{ GLM } & \multicolumn{2}{c}{ Global average } \\
\hline & HbO & HbR & HbO & HbR & HbO & HbR \\
& \multicolumn{2}{c}{$\begin{array}{c}t \text {-value } \\
\text { Mean concentration changes }(\mu \mathrm{M}) \pm \text { standard deviation }\end{array}$} \\
\hline Broca & $4.89^{*}$ & $-4.89^{*}$ & $5.88^{*}$ & $-5.88^{*}$ & $6.11^{*}$ & $-6.11^{*}$ \\
Left & 0.09 & -0.02 & 0.04 & -0.01 & 0.97 & -0.19 \\
& \pm 0.22 & \pm 0.04 & \pm 0.09 & \pm 0.02 & \pm 1.97 & \pm 0.29 \\
\hline Broca & $3.91^{*}$ & $-3.91^{*}$ & $2.91^{*}$ & $-2.91^{*}$ & $3.24^{*}$ & $-3.24^{*}$ \\
Right & 0.06 & -0.01 & 0.02 & -0.003 & 0.39 & -0.08 \\
& \pm 0.17 & \pm 0.03 & \pm 0.06 & \pm 0.01 & \pm 1.48 & \pm 0.29 \\
\hline
\end{tabular}

Note. Relative contribution of the two channels in the left Broca's area as defined by Brodmann 44, 45, and the homologous channels in the right hemisphere was evaluated using mean relative weights for the PARAFAC component, mean $\beta_{1}$ values for the HRF from the GLM, and global average for concentration changes. For each method, mean relative contribution during the 30 s of the verbal fluency task was tested against 0 by using a onesample t-test.

*One-tail $p<0.05$.

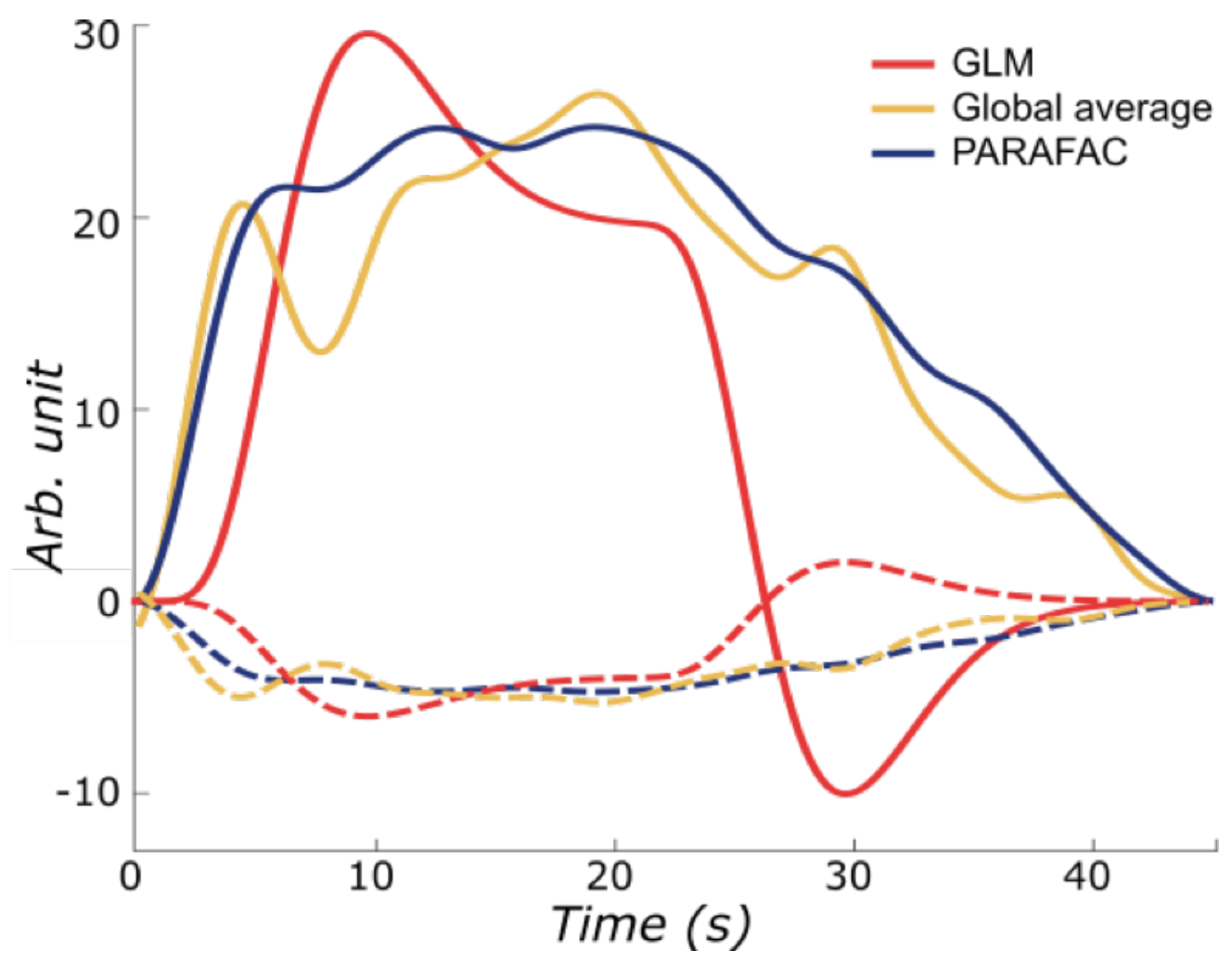

Fig. 5 Description of the task-related brain activation pattern: Representative time course of hemodynamic response in the left Broca's area during the 30-s verbal fluency task, as identified with the PARAFAC temporal signature (blue line), modeled HRF as estimated by GLM (red line), and global averaged channels (yellow line). Solid lines indicate concentration changes in $\mathrm{HbO}$, dashed lines concentration changes in $\mathrm{HbR}$, respectively. 


\section{Discussion}

This study aimed to introduce and validate parallel factor analysis (PARAFAC), a multidimensional decomposition technique, for the analysis of fNIRS data. First, we investigated the efficacy of PARAFAC for the detection and correction of movement artifacts. Second, we explored the usefulness of PARAFAC in identifying the dominant task-related activation in noisy experimental data. For both parts of our intended validation, results were compared to commonly used methods in fNIRS.

\subsection{Artifact detection and correction}

Several studies have shown promising results by using PARAFAC for artifact detection and correction, as well as to extract patterns of cerebral activation in EEG data ${ }^{11,12,15}$. Our results are in line with those studies, thus extending the application of PARAFAC for artifact correction and the extraction of cerebral activation patterns in fNIRS.

Signal quality was evaluated using the SNR for segments in which artifacts were detected. All artifact correction techniques resulted in a significant improvement of the signal's quality, and thus a decrease of the noise in the artifact segments. ICA was applied, as reported in the literature, to the entire signal ${ }^{27}$, while tPCA and PARAFAC were target-based, i.e. only decomposing the signal during specific segments where artifacts had been detected. In line with previous findings, our analyses revealed an advantage in applying target corrections (tPCA and PARAFAC) instead of whole-block (ICA) correction, resulting in a better signal quality ${ }^{23,24}$. Furthermore, comparisons between TPCA and PARAFAC suggest that the multidimensional approach of PARAFAC results in a slightly better correction. This means concentration changes in artifact segments after PARAFAC corrections achieved the smallest variance among the three methods. Nevertheless, it remains important to consider that the difference observed between IPCA and PARAFAC, though significant, has a small effect size, while the efficacy of PARAFAC is more robust as compared to ICA (large effect size). As proposed by Yücel and colleagues ${ }^{24}$, target artifact correction prevents large changes in the overall composition of the signal, and exclusively corrects the noisy time interval of the artifact. It allows the decomposition to clearly sort a component representing the artifact signature, without considering the characteristics of these channels during the intervals without artifacts. Target decomposition techniques are thus beneficial for a precise identification of the artifact's signatures.

Signal quality was also evaluated using Pearson's correlation between the time course of both wavelengths, based on the assumption that different wavelengths (related to $\mathrm{HbO}$ and $\mathrm{HbR}$ concentrations) assessed at the same location are similarly affected by movement artifacts ${ }^{17}$. Thus, the signal shows higher correlation between wavelengths when it reflects the same movement artifact, compared to a lower correlation for intervals where less or no artifact is present. Of the three different decomposition techniques, correction with PARAFAC led to the lowest correlation between $\mathrm{HbO}$ and $\mathrm{HbR}$ indices, as well as the correlation index that most resembled the nonartifactual resting-state baseline reference. Even though the resting-state signal has been reported to show slightly different hemodynamic changes, compared to the task-stimulation ${ }^{18,57}$, we consider it a suitable reference for a time interval without artifacts, because it should not be affected by articulation. Two-dimensional decomposition on the other hand, such as ICA and tPCA, analyzes both wavelengths as independent measures of the fNIRS signal, even though they are in fact highly related, since they are acquired at the same location ${ }^{17}$. 
Taken together, our results suggest that decomposition with PARAFAC allows an accurate detection and correction of artifacts. Decomposition with PARAFAC offers an approach whereby it is rather easy to make a selection of relevant components that differentiate between signatures related to artifacts or other relevant characteristics of the signal. Its efficacy resembles that obtained from tPCA, but its consideration of the intrinsically multidimensional structure of the fNIRS signal, as well as the absence of orthogonality or independence constraints, stand out as important advantages of PARAFAC. Even if fNIRS is considered to be less sensitive to movements, compared to other neuroimaging techniques such as fMRI, appropriate tools for artifact correction are essential, particularly for data acquired in populations where cooperation is limited, such as children and clinical populations ${ }^{58}$. PARAFAC thus appears to be a suitable alternative to the currently used approaches, and will be a valuable add-on for fNIRS studies and clinical examinations by minimizing the amount of non-usable data and improving data quality.

\subsection{Characterizing fNIRS activation patterns}

We applied a PARAFAC model with one single component, because by using the shortdistance channel, we corrected our signal for systematic influences of physiology, and assumed that the remaining dominant signature represented the task-related cerebral activation. 2D decomposition have previously been used as an alternative to estimate the contribution of physiology to the fNIRS signal ${ }^{59}$. So potentially, PARAFAC could be considered for such a correction as well. Nevertheless, we choose to maintain the same standard procedure to address physiological noise across all three conditions (i.e. GLM, global average and PARAFAC). The use of short-distance channels is considered a valuable and reliable measure for such correction ${ }^{60}$. Furthermore, the component extracted from PARAFAC is coherent with the current literature. We used a semantic verbal fluency paradigm that has consistently elicited cerebral activation within brain regions commonly associated with expressive language, namely the left Broca's area ${ }^{36-38}$. Therefore, we defined the ROIs as the left Broca's area, as well as the homologous areas in the right hemisphere. We expected that the temporal and hemodynamic distributions would be reflected as an increase of $\mathrm{HbO}$ and a slight dip of $\mathrm{HbR}$ within these regions roughly 5 seconds after task onset ${ }^{50}$.

The modeled time course indicates that both data-driven approaches, PARAFAC and global average, reveal an earlier onset of the hemodynamic response in contrast to GLM. This illustrates how a data-driven approach provides more flexibility particularly in paradigms or populations where cerebral activation may show alterations, thus does not follow a modelled time course. The three approaches revealed significant hemodynamic responses within the ROI. However, topographical representations of the task-related activation do not clearly differ between GLM and global average, while PARAFAC provides a slightly more distributed activation pattern. Since the GLM imposes a modeled HRF with a specific time course, it may exclude channels with minor temporal deviations, an aspect that has been discussed critically on several previous studies ${ }^{56,57}$. Global average intrinsically represents an approach to both minimizing variability and outlining the most constant information from the data, hence it is not surprising to obtain a more condensed spatial pattern. Both GLM and global average likely show the most dominant activation representing an over-simplified pattern of cerebral activation. Topographical findings from PARAFAC revealed a brain activation that spreads into more cerebral regions known to be involved in expressive language processing ${ }^{58,59,60}$.

What is more, given that neither GLM nor global average treats $\mathrm{HbO}$ and $\mathrm{HbR}$ simultaneously, their results may only demonstrate strong concentration changes in either 
dimension, while ignoring the task-related dynamics of $\mathrm{HbO}$ and $\mathrm{HbR}$. However, PARAFAC components are based on matrices that simultaneously consider time, space and hemodynamic dimensions, thus resulting in a component that considers the dynamics of both. Several studies have emphasized the importance of including both indices of concentration changes, in order to reliably interpret hemodynamic changes ${ }^{32,33,35}$. Applying PARAFAC for data analysis in fNIRS would allow a better exploitation of both $\mathrm{HbO}$ and $\mathrm{HbR}$ indices and their dynamics, hence leading to enriched results.

\subsection{Usefulness and limitations of PARAFAC}

PARAFAC is a data-driven approach based on the linear relations of the three dimensions of the fNIRS signal ${ }^{5,11,12}$. PARAFAC has the advantage of allowing for the three-way arrays of data to be uniquely decomposed into a sum of components, each of which is a trilinear combination of factors or signatures. One of the only statistical requirements of PARAFAC is that of a moderate linear independence across components, i.e. their time course, topography and wavelength characteristics. This is a less stringent requirement than previous models that underly space/time decompositions (PCA or ICA). Each component provides characteristics of a particular pattern identified within the mixed measured fNIRS signal. When there are empirical or theoretical reasons to expect more than one relevant component (e.g. resting state functional connectivity analysis, epileptic activity, physiological aspects, etc.), PARAFAC would also allow to disentangle between several components.

Different from ICA, PARAFAC components can be ordered according to their importance in explaining the data's variance ${ }^{62}$. This is why many studies using ICA apply data reduction or clustering techniques such as PCA prior to the execution of ICA $^{27,66}$. Moreover, when using PARAFAC, the description of the data is based on more dimensions, and with less theoretical constraints (orthogonality, independence) which lead to the identification of fewer relevant components ${ }^{11,12,67,68}$. Compared to ICA, the selection of the relevant components is thus simplified. Importantly, when using PARAFAC, it is not recommended to ask for more than five components, as this increases the risk of overfitting the data with the decomposition model. Usually, three to five components sufficiently describe the signal's relevant characteristics ${ }^{14}$. Similar to PCA, a typical choice made when using PARAFAC analysis, is that of ordering the extracted components according to their contribution to explaining the variance of the data. In this sense, it can be expected that the first PARAFAC component will always represent the highest activity, which, in the case of target artifact correction, will correspond to the artifact activity. This makes PARAFAC also a promising choice to explore the development of simple automatic methodologies for the detection and correction of such artifacts.

Furthermore, as GLM and global averaging treat each channel independently, these techniques provide a narrower spatial representation of the dominant activation. PARAFAC analysis suggests a rather distributed activation pattern, while still revealing a dominant activation within our ROI covering Broca's area. This strongly corresponds to the current understanding of cerebral processing, whereby large scale networks, and not isolated regions, are involved in language processing ${ }^{58,59,60}$. The use of PARAFAC to characterize hemodynamic patterns therefore appears to be more suitable for reflecting the cerebral processes occurring in distributed networks, rather than for the identification of a specific core region. Moreover, the topographic signature correspond to a whole time course of the extracted activations, and can easily be subjected to diffusion optical tomography in order to locate the concentration changes in $\mathrm{HbR}$ and $\mathrm{HbO}$ in the brain cortex ${ }^{69}$. 
Some challenges of PARAFAC have already been discussed in previous studies ${ }^{11,12}$. First, PARAFAC assumes linear relations of the temporal characteristics between different channels. Despite being one of the most common and simple models, imposing linearity may not entirely reflect complex cerebral processes ${ }^{70}$. Some attempts have been made to introduce approaches that tolerate non-linear relations ${ }^{71,72}$. However, to date, linear models remain the most popular approach for analysis of macroscopic data in neuroscience. Thus, it represents a general limit of the domain, rather than one specific to PARAFAC.

A second limitation regards the need for careful preprocessing of data before characterizing brain activity. When PARAFAC was introduced for the analysis of EEG data, the authors emphasized the importance of searching for constant factors, outliers, and degeneracy ${ }^{9,12}$. Detailed information on how to deal with these aspects can be found in previous literature ${ }^{12}$. In the current study, the implementation of PARAFAC into the LIONNIRS toolbox, gave us a certain flexibility regarding the channels and time segments to be included. Thus, we were able to not consider deviant channels when using PARAFAC.

Finally, an application of PARAFAC, not yet tested in fNIRS, is its use as a screening tool. For instance, Miwakeichi and colleagues ${ }^{12}$ applied PARAFAC to an EEG dataset in order to extract one component related to ocular movement artifacts, and subsequently used algorithms based on that component to screen a second dataset in order to identify and correct similar artifacts. Although this can be useful for correcting artifacts that have consistent topographical and wavelength profiles, movement artifacts were mostly related to articulation in our study, often showing quite different signatures. A different paradigm may enable the testing of this application of PARAFAC in fNIRS.

Our findings also encourage the general application of PARAFAC for multidimensional decomposition in neuroimaging, where data can often be described on more than two dimensions. Beyond the obvious dimensions of a technique such as that of time $\times$ space $\times$ wavelength (fNIRS) or frequency (EEG), characteristics of the task paradigm or group variables could also be considered in the decomposition mode ${ }^{15}$. For instance, if a "group" dimension was to be included, groups could be compared regarding their relative weight over the main cerebral activation component that would have been outlined from the PARAFAC model.

\subsection{Limits of the current study}

The use of a language paradigm allowed for the induction of artifacts that were mainly related to articulation, which limits the generalization from our results. From video recordings, however, we were able to acknowledge that swallowing, jaw or tongue movements, eye blinks, and frowning also induced artifacts in our dataset. Further fNIRS studies using PARAFAC, and including different task paradigms and resting-state, could show its utility for a wider range of artifacts. The validation of PARAFAC for artifact correction was conducted by comparing results with two other decomposition techniques. This could be considered one limit of the present study, because we did not consider other correction approaches for comparison. The objective of this study was not to conduct exhaustive and systematic comparisons in order to identify the best method for artifact correction, but rather to introduce PARAFAC as an alternative and adequate method in fNIRS analysis. Future studies could therefore expand the validation of PARAFAC and extend comparisons of its performance to other tools that do not necessarily use decomposition, such as spline interpolation or wavelet filtering. With relation to that, even if there were to be a slight advantage of PARAFAC over tPCA in artifact correction results, the small difference might not have a concrete impact on the applicability of those two methods. Qualitative differences 
between IPCA and PARAFAC regarding the type of artifacts and corrected signal should be further assessed and described in subsequent studies to better understand how their efficacy may vary according to the artifact's characteristics.

Finally, another limitation related to the generalization of our results might arise from the specific use of the simple least square technique to implement PARAFAC. Other methods have been proposed for estimating PARAFAC models, but some studies have shown that, given the uniqueness of the solution, they do not usually outperform the simple least square technique ${ }^{73}$.

\section{Conclusion}

PARAFAC allows for the simultaneous treatment of both wavelengths or $\mathrm{HbO}$ and $\mathrm{HbR}$ during fNIRS data analyses. Our findings validate previous results in EEG data and promote multidimensional PARAFAC decomposition as a promising new tool for the extraction of relevant signatures represented in the fNIRS signal. The validation of its use for the correction of movement artifacts, as well as to extract the pattern of a task-related cerebral activation, paves the way for its future use in fNIRS research.

\section{Disclosures}

We have nothing to disclose.

\section{Acknowledgments/Funding sources}

The authors warmly thank all participants of the study, as well as Catherine Bernard, Kathy Martel and Solène Fourdain for their support during data acquisition.

This work was funded by the National Science and Engineering Research Council of Canada (NSERC) (\#2015-04199 and \# CGSD3-518503-2018); the Canada Research Chairs (\#950-232661); the Ministère des Affaires internationales et de la Francophonie du Québec; the Fonds de Recherche du Québec Santé (FRQS, \#28811 and \#35450); the Fonds de recherche du Québec - Nature et technologies (FRQNT, \#255473); and the SickKids Foundation (\#NI16-058). 


\section{Appendix}

Case 1

$\overline{\sum_{t=1}^{n} A}(\mathrm{t})>0$ and $\mathrm{C} \mathrm{HbO}>\mathrm{HbR}$

$1 \times 1 \times 1=1$

$\mathrm{A}=\mathrm{A} ; \mathrm{B}=\mathrm{B} ; \mathrm{C}=\mathrm{C}$

Case 2

$\overline{\sum_{t=1}^{n} A(\mathrm{t})}<0$ and $\mathrm{C} \mathrm{HbO}>\mathrm{HbR}$

$-1 \times-1 \times 1=1$

$\mathrm{A}=-\mathrm{A} ; \mathrm{B}=-\mathrm{B} ; \mathrm{C}=\mathrm{C}$

\section{A $=$ Temporal signature $\mathbf{B}=$ Spatial signature}
11
12
13
14
15
16
17
18
19

\section{$\mathrm{C}=$ Hemodynamic signature}

Fig. 6 Sign adjustment for PARAFAC components: Force $\mathrm{HbO} / \mathrm{HbR}$ responses to be positive/negative by changing the sign of pairs of signatures extracted. This is due to the trivial sign indeterminacy as the trilinear multiplication in PARAFAC gives the same results for $1 \times 1 \times 1$ as for $1 \times(-1) \times(-1)$ so it does not discriminate between these two options in the decomposition.

\section{References}

1. F. F. Jobsis, "Noninvasive, infrared monitoring of cerebral and myocardial oxygen sufficiency and circulatory parameters," Science 198(4323), 1264-1267, American Association for the Advancement of Science (1977) [doi:10.1126/SCIENCE.929199].

2. S. Brigadoi et al., "Motion artifacts in functional near-infrared spectroscopy: A comparison of motion correction techniques applied to real cognitive data," Neuroimage 85, 181-191 (2014) [doi:10.1016/j.neuroimage.2013.04.082].

3. R. J. Cooper et al., "A systematic comparison of motion artifact correction techniques for functional near-infrared spectroscopy," Front. Neurosci. 6(OCT), 1-10 (2012) [doi:10.3389/fnins.2012.00147].

4. S. Tak and J. C. Ye, "Statistical analysis of fNIRS data: A comprehensive review," Neuroimage 85, 72-91 (2014) [doi:10.1016/j.neuroimage.2013.06.016].

5. R. Bro, "Multi-way Analysis in the Food Industry Models, Algorithms, and Applications," Royal Veterinary and Agricultural University (1998).

6. R. A. Harshman, "Foundations of the PARAFAC procedure: Models and conditions for an 'explanatory' multimodal factor analysis," in UCLA Working Papers in Phonetics 16, University Microfilms, Ann Arbor, Michigan (1970).

7. J. D. Carroll and J.-J. Chang, "Analysis of individual differences in multidimensional scaling via an n-way generalization of 'Eckart-Young' decomposition," Psychometrika 35(3), 283-319 (1970) [doi:10.1007/BF02310791].

8. L. R. Tucker, "Some mathematical notes on three-mode factor analysis," Psychometrika 31(3), 279-311 (1966). 
9. A. S. Field and D. Graupe, "Topographic component (Parallel Factor) analysis of multichannel evoked potentials: Practical issues in trilinear spatiotemporal decomposition," Brain Topogr. 3(4), 407-423 (1991) [doi:10.1007/BF01129000].

10. J. Möcks, "Decomposing event-related potentials: A new topographic components model," Biol. Psychol. 26(1-3), 199-215 (1988) [doi:10.1016/0301-0511(88)90020-8].

11. E. Martínez-Montes et al., "Concurrent EEG/fMRI analysis by multiway Partial Least Squares,” Neuroimage 22(3), 1023-1034 (2004) [doi:10.1016/j.neuroimage.2004.03.038].

12. F. Miwakeichi et al., "Decomposing EEG data into space-time-frequency components using Parallel Factor Analysis," Neuroimage 22(3), 1035-1045 [doi:10.1016/j.neuroimage.2004.03.039].

13. V. Calhoun, "Data-driven approaches for identifying links between brain structure and

14. E. Acar et al., "Multiway analysis of epilepsy tensors," Bioinformatics 23(13), i10-i18 (2007) [doi:10.1093/bioinformatics/btm210].

15. M. Mørup et al., "Parallel Factor Analysis as an exploratory tool for wavelet transformed event-related EEG," Neuroimage 29(3), 938-947 [doi:10.1016/j.neuroimage.2005.08.005].

16. S. Lloyd-Fox, A. Blasi, and C. E. Elwell, "Illuminating the developing brain: The past, present and future of functional near infrared spectroscopy," Neurosci. Behav. Rev. 34, 269284 (2010) [doi:10.1016/j.neubiorev.2009.07.008].

17. X. Cui, S. Bray, and A. L. Reiss, "Functional near infrared spectroscopy (NIRS) signal improvement based on negative correlation between oxygenated and deoxygenated hemoglobin dynamics," Neuroimage 49(4), 3039-3046 (2010) [doi:10.1016/j.neuroimage.2009.11.050].

18. H. Zhang et al., "Is resting-state functional connectivity revealed by functional near-infrared spectroscopy test-retest reliable?," J. Biomed. Opt. 16(6), 067008 (2011) [doi:10.1117/1.3591020].

19. G. James et al., "Classification," in An Introduction to Statistical Learning, pp. 127-173, Springer, New York, NY (2013) [doi:10.1007/978-1-4614-7138-7_4].

20. X. Zhang et al., "Signal processing of functional NIRS data acquired during overt speaking," Neurophotonics 4(04), 1 (2017) [doi:10.1117/1.NPh.4.4.041409].

21. K. Peng et al., "fNIRS-EEG study of focal interictal epileptiform discharges," Epilepsy Res. 108, 491-505 (2014) [doi:10.1016/j.eplepsyres.2013.12.011].

22. H. Becker et al., "Multi-way space-time-wave-vector analysis for EEG source separation," Signal Processing 92, 1021-1031 (2011) [doi:10.1016/j.sigpro.2011.10.014].

23. H. F. Behrendt et al., "Motion correction for infant functional near-infrared spectroscopy with an application to live interaction data," Neurophotonics 5(01), 1 (2018) [doi:10.1117/1.NPh.5.1.015004]. 
24. M. A. Yücel et al., "Targeted principle component analysis: A new motion artifact correction approach for near-infrared spectroscopy,” J. Innov. Opt. Health Sci. 7(2), 1350066 (2014) [doi:10.1142/S1793545813500661].

25. M. A. Kamran, M. M. N. Mannan, and M. Y. Jeong, "Cortical Signal Analysis and Advances in Functional Near-Infrared Spectroscopy Signal: A Review," Front. Hum. Neurosci. 10, 261 (2016) [doi:10.3389/fnhum.2016.00261].

26. I. Tachtsidis and F. Scholkmann, "False positives and false negatives in functional nearinfrared spectroscopy: issues, challenges, and the way forward," Neurophotonics 3(3), 031405 (2016) [doi:10.1117/1.NPh.3.3.031405].

27. H. Zhang et al., "Functional connectivity as revealed by independent component analysis of resting-state fNIRS measurements," Neuroimage 51, 1150-1161 (2010) [doi:10.1016/j.neuroimage.2010.02.080].

28. A. Jung et al., "Fastgeo-a histogram based approach to linear geometric ICA," in Proceedings of ICA Vol. 1, pp. 349-354 (2001).

29. P. Comon, "Independent component analysis, A new concept?," Signal Processing 36(3), 287-314 (1994) [doi:10.1016/0165-1684(94)90029-9].

30. C. Jutten and J. Herault, "Blind separation of sources, part I: An adaptive algorithm based on neuromimetic architecture," Signal Processing 24(1), 1-10 (1991) [doi:10.1016/01651684(91)90079-X].

31. D. A. Boas et al., "The accuracy of near infrared spectroscopy and imaging during focal changes in cerebral hemodynamics," Neuroimage 13(1), 76-90 (2001) [doi:10.1006/nimg.2000.0674].

32. H. Obrig et al., "From acoustic segmentation to language processing: evidence from optical imaging," Front. Neuroenergetics 2(13), 13 (2010) [doi:10.3389/fnene.2010.00013].

33. V. Quaresima, S. Bisconti, and M. Ferrari, "A brief review on the use of functional nearinfrared spectroscopy (fNIRS) for language imaging studies in human newborns and adults," Brain Lang. 121(2), 79-89 (2012) [doi:10.1016/j.bandl.2011.03.009].

34. M. L. Schroeter et al., "Investigating the post-stimulus undershoot of the BOLD signal-a simultaneous fMRI and fNIRS study," Neuroimage 30(2), 349-358 (2006) [doi:10.1016/J.NEUROIMAGE.2005.09.048].

35. M. Wolf and G. Greisen, "Advances in near-infrared spectroscopy to study the brain of the preterm and term neonate," Clin. Perinatol. 36(4), 807-834 (2009) [doi:10.1016/j.clp.2009.07.007].

36. W. D. Gaillard et al., "Developmental aspects of language processing: fMRI of verbal fluency in children and adults," Hum. Brain Mapp. 18(3), 176-185 (2003) [doi:10.1002/hbm.10091].

37. N. Paquette et al., "Developmental patterns of expressive language hemispheric lateralization in children, adolescents and adults using functional near-infrared spectroscopy," Neuropsychologia $\quad \mathbf{6 8}, \quad 117-125$ [doi:10.1016/j.neuropsychologia.2015.01.007]. 
38. A. Gallagher, J. Tremblay, and P. Vannasing, "Language mapping in children using restingstate functional connectivity: comparison with a task-based approach," J. Biomed. Opt. 21(12), 125006 (2016) [doi:10.1117/1.JBO.21.12.125006].

39. G. H. Klem et al., "The ten-twenty electrode system of the International Federation," Electroencephalogr Clin Neurophysiol 52(3), 3-6 (1999).

40. S. Brigadoi and R. J. Cooper, "How short is short? Optimum source-detector distance for short-separation channels in functional near-infrared spectroscopy," Neurophotonics 2(2), 025005 (2015) [doi:10.1117/1.NPh.2.2.025005].

41. F. Scarpa et al., "A reference-channel based methodology to improve estimation of eventrelated hemodynamic response from fNIRS measurements," Neuroimage 72, 106-119 (2013) [doi:10.1016/j.neuroimage.2013.01.021].

42. A. Villringer and U. Dirnagl, "Coupling of brain activity and cerebral blood flow: basis of functional neuroimaging," Cerebrovasc. Brain Metab. Rev. 7(3), 240-276 (1995).

43. A. Aarabi and T. J. Huppert, "Characterization of the relative contributions from systemic physiological noise to whole-brain resting-state functional near-infrared spectroscopy data using single-channel independent component analysis," Neurophotonics 3(2), 025004 (2016) [doi:10.1117/1.NPh.3.2.025004].

44. M. Plank, "Ocular correction ICA,” Brain Prod. Press Release 49 (2013).

45. C. A. Andersson and R. Bro, "The N-way Toolbox for MATLAB," Chemom. Intell. Lab. Syst. 52(1), 1-4 (2000) [doi:10.1016/S0169-7439(00)00071-X].

46. L. Kocsis, P. Herman, and A. Eke, "The modified Beer-Lambert law revisited," Phys. Med. Biol. 51(5), N91-N98 (2006) [doi:10.1088/0031-9155/51/5/N02].

47. K. J. Friston et al., Statistical Parametric Mapping: The Analysis of Functional Brain Images, Academic Press, London, UK (2011).

48. M. L. Schroeter et al., "Towards a standard analysis for functional near-infrared imaging," Neuroimage 21(1), 283-290 (2004) [doi:10.1016/j.neuroimage.2003.09.054].

49. Y. Minagawa-Kawai et al., "Optical brain imaging reveals general auditory and languagespecific processing in early infant development," Cereb. Cortex 21(2), 254-261 (2011) [doi:10.1093/cercor/bhq082].

50. G. H. Glover, "Deconvolution of Impulse Response in Event-Related BOLD fMRI1," Neuroimage 9(4), 416-429 (1999) [doi:10.1006/nimg.1998.0419].

51. A. P. Holmes and K. J. Friston, "Generalisability, Random Effects \&amp; Population Inference,” Neuroimage 7(4), S754 (1998) [doi:10.1016/S1053-8119(18)31587-8].

52. M. Monti, "Statistical Analysis of fMRI Time-Series: A Critical Review of the GLM Approach,” Front. Hum. Neurosci. 5(28), 1-13 (2011) [doi:10.3389/fnhum.2011.00028].

53. M. Uga et al., "Optimizing the general linear model for functional near-infrared spectroscopy: an adaptive hemodynamic response function approach," Neurophotonics 1(1), 015004 (2014) [doi:10.1117/1.NPh.1.1.015004]. 
54. R. Bro and S. De Jong, "A fast non-negativity-constrained least squares algorithm," J. Chemom. 11(5), 393-401 (1997)

[doi:10.1002/(SICI)1099128X(199709/10)11:5<393::AID-CEM483>3.0.CO;2-L].

172

173

174

175

176

177

178

179

180

181

182

183

184

185

55. K. T. Sweeney et al., "A Methodology for Validating Artifact Removal Techniques for Physiological Signals,” IEEE Trans. Inf. Technol. Biomed. 16(5), 918-926 (2012) [doi:10.1109/TITB.2012.2207400].

56. J. Cohen, Statistical Power Analysis for the Behavioral Sciences, Second, Lawrence Erlbaum Associates, Inc., New York, NY (1988).

57. T. T. Liu, "Neurovascular factors in resting-state functional MRI," Neuroimage 80, 339348 (2013) [doi:10.1016/J.NEUROIMAGE.2013.04.071].

58. S. Tak et al., "Dynamic causal modelling for functional near-infrared spectroscopy," Neuroimage 111, 338-349 (2015) [doi:10.1016/j.neuroimage.2015.02.035].

59. G. Jasdzewski et al., "Differences in the hemodynamic response to event-related motor and visual paradigms as measured by near-infrared spectroscopy," Neuroimage 20(1), 479-488, Academic Press Inc. (2003) [doi:10.1016/S1053-8119(03)00311-2].

60. T. Sato et al., "Reduction of global interference of scalp-hemodynamics in functional nearinfrared spectroscopy using short distance probes," Neuroimage 141, 120-132 (2016) [doi:10.1016/j.neuroimage.2016.06.054].

61. F. Scarpa et al., "A GLM-Based Approach to Estimate Stimulus- Evoked Hemodynamic Response from fNIRS Measurements," in 6th International Conference on Bioinformatics and Biomedical Engineering, p. iCBBE 2012 (2012).

62. D. Poeppel et al., "Towards a New Neurobiology of Language," J. Neurosci. 32(41), 1412514131 (2012) [doi:10.1523/JNEUROSCI.3244-12.2012].

63. G. Hickok and D. Poeppel, "Towards a functional neuroanatomy of speech perception," Trends Cogn. Sci. 4(4), 131-138 (2000) [doi:10.1016/S1364-6613(00)01463-7].

64. M. A. Skeide and A. D. Friederici, "The ontogeny of the cortical language network," Nat. Rev. Neurosci. 17(5), 323-332 (2016) [doi:10.1038/nrn.2016.23].

65. J. Y. Choi, H. Hwang, and M. E. Timmerman, "Functional Parallel Factor Analysis for Functions of One- and Two-dimensional Arguments," Psychometrika 83(1), 1-20 (2018) [doi:10.1007/s11336-017-9558-9].

66. S. Makeig, "Dynamic Brain Sources of Visual Evoked Responses," Science (80-. ). 295(5555), 690-694 (2002) [doi:10.1126/science.1066168].

67. N. D. Sidiropoulos and R. Bro, "On the uniqueness of multilinear decomposition of N-way arrays," J. Chemom. 14(3), 229-239 (2000) [doi:10.1002/1099128X(200005/06)14:3<229::AID-CEM587>3.0.CO;2-N].

68. W. Deburchgraeve et al., "Neonatal seizure localization using PARAFAC decomposition," Clin. Neurophysiol. 120(10), 1787-1796 (2009) [doi:10.1016/j.clinph.2009.07.044].

69. J. Tremblay et al., "Comparison of source localization techniques in diffuse optical tomography for fNIRS application using a realistic head model," Biomed. Opt. Express 9(7), 448-454 (2018) [doi:10.1364/BOE.9.002994]. 
209 70. T. J. Huppert, "Commentary on the statistical properties of noise and its implication on general linear models in functional near-infrared spectroscopy," Neurophotonics 3(1), 010401 (2016) [doi:10.1117/1.NPh.3.1.010401].

212 71. M. Hu and H. Liang, “A copula approach to assessing Granger causality,” Neuroimage 100, 125-134 (2014) [doi:10.1016/j.neuroimage.2014.06.013].

214 72. W. A. Freiwald et al., "Testing non-linearity and directedness of interactions between neural groups in the macaque inferotemporal cortex," J. Neurosci. Methods 94(1), 105-119 (1999) [doi:10.1016/S0165-0270(99)00129-6].

73. N. M. Faber, R. Bro, and P. K. Hopke, "Recent developments in CANDECOMP/PARAFAC algorithms: A critical review," in Chemometrics and Intelligent Laboratory Systems 65(1), pp. 119-137 (2003) [doi:10.1016/S0169-7439(02)00089-8]. 